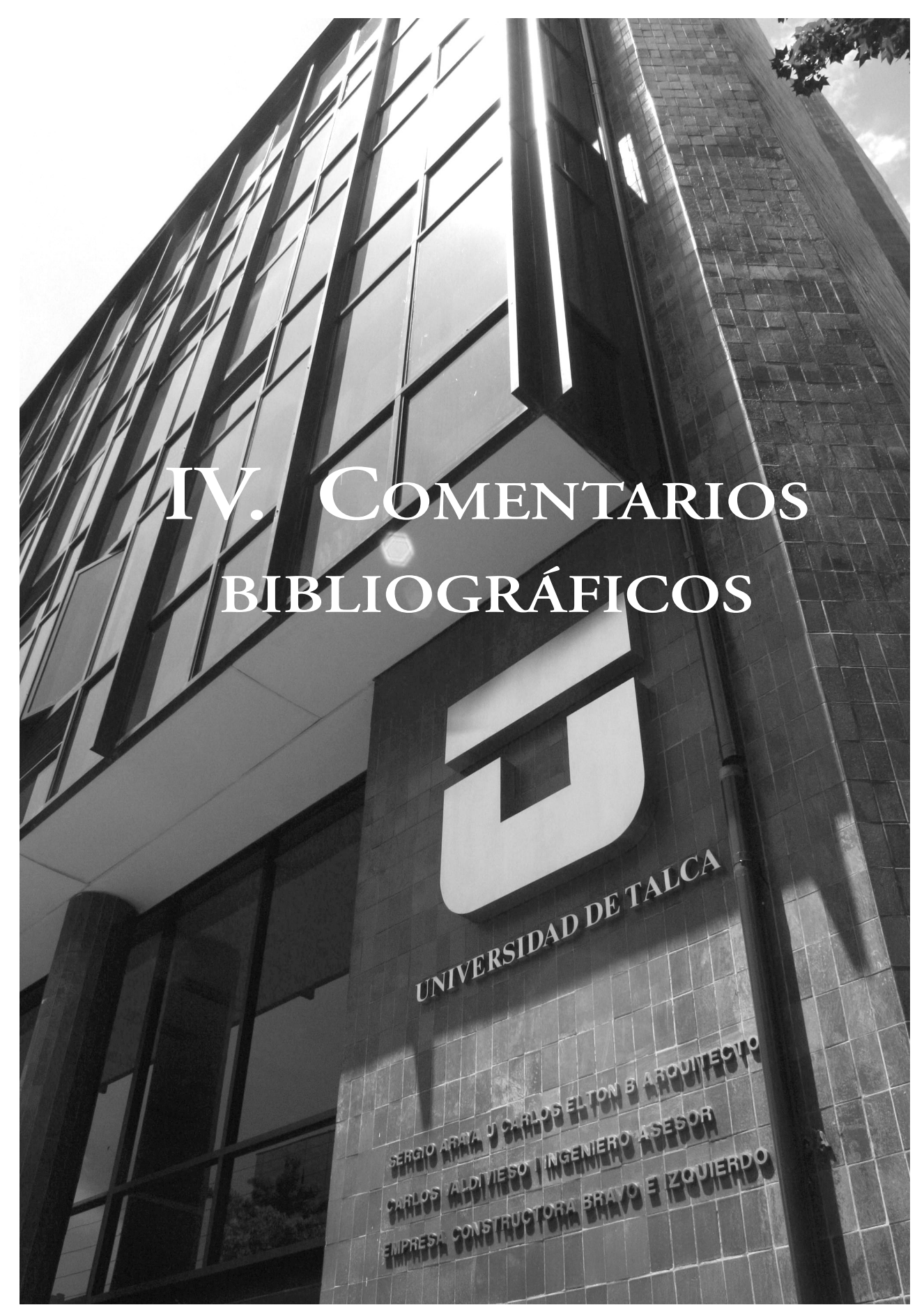



Estudios Constitucionales, Año 11, No 2, 2013, pp. 785 - 792.

ISSN 07180195

Centro de Estudios Constitucionales de Chile Universidad de Talca

"E possibile una legalità globale? Il rule of law e la governance del mondo, Palombella, Gianluigi (2012). Bologna, Il Mulino, 272 pp.”

Susy Muñoz Merkle

\section{È POSSIBILE UNA LEGALITÀ GLOBALE? IL RULE OF LAW E LA GOVERNANCE DEL MONDO, PALOMBELLA, GIANLUIGI (2012)}

Bologna, Il Mulino, 272 Pp.*

SusY Muñoz MerKLE** Universidad de Valparaíso - Chile susymunoz26@hotmail.com

Este libro aborda el análisis jurídico de la posibilidad de un derecho planetario. Esto implica diversas imbricaciones de lo jurídico con otros aspectos de la realidad mundial, y de otras disciplinas, destacando el subtítulo las cercanas relaciones políticas ${ }^{1}$, aun cuando la perspectiva es predominantemente de filosofía del derecho. El autor es profesor en la Universidad de Parma y ha escrito otros libros de gran impacto. Cabe destacar, el libro titulado: "Relocating the rule of law" (Relocalizando el imperio del derecho), escrito en coautoría con N. Walker, editado por la casa editorial Hart en el año 2009. Esta coautoría es importante porque para Palombella la tradición jurídica anglosajona es particularmente relevante, en el análisis de una posible legalidad global, tal como se desprende del subtítulo de este libro. El profesor Palombella ha centrado su interés, como suele ser la tónica en los grandes centros de estudios públicos europeos, en la emergencia de un derecho global y en el examen de sus principales rasgos. ${ }^{2}$

El libro del profesor Palombella tiene 272 páginas, está dividido en cinco capítulos y un apéndice, este último, a mi parecer, debió ser el capítulo sexto, ya que

\footnotetext{
* Trabajo recibido el 3 de abril y aprobado el 17 de julio de 2013.

** Magíster en Gestión Jurídica de las Empresas de la Universidad de Valparaíso; Alumna de Programa de Doctorado en Derecho, con Mención en Derecho Público de la misma Universidad; Profesora de la Escuela de Derecho de la Universidad de Valparaíso.

1 En Chile y desde una perspectiva politológica, Ernesto Ottone publicó su libro Gobernar la Globalización (Ediciones UDP, Santiago, 2011).

2 En este sentido, otros libros del autor sobre esta temática son: La autoridad de los derechos (L'autorità dei diritti, Laterza, 2002); Relocalizando el imperio del derecho. (Relocating the rule of law, con N. Walker; Hart Publishing, 2009); Imperio del Derecho y Democracia. (Rule of law and democracy (con L. Morlino; Brill, 2010). También interesantes son sus obras relacionadas con este tópico: Después la certeza (Dopo la certeza, Dedalo, 2006).
} 
no se trata de anexos, sino que propiamente de un análisis general y conclusivo. El primer capítulo aborda el ideal de la legalidad y el Rule of law. El segundo capítulo se refiere a otros estados de la cuestión del imperio de la ley, considerado el vocablo ley en sentido amplio. Compara el rol del Derecho Internacional con el de los Estados con el del imperio de la ley o del derecho, relaciones, implicancias y problemas; cuestiona si el imperio de la ley puede ser universal y unilateral; distingue niveles de aplicación o cumplimiento del imperio de la ley; plantea la posibilidad de considerar el imperio de la ley como una tercera vía y analiza la práctica del reconocimiento basada en el marco teórico hartiano. El tercer capítulo describe los distintos derechos que pueblan el mundo, en el sentido de profundizar el concepto de multinivel de legalidad y de cómo estos diferentes niveles interactúan (derechos subestatales, estatales, regionales, internacionales, supranacionales y globales), utilizando para este efecto la metáfora de los estratos geológicos. El cuarto capítulo profundiza el problema que implica un imperio de la ley global o mundial especialmente en cuanto a la interacción entre el derecho interno y externo a la luz de la teoría del derecho, poniendo énfasis en la tensión entre la justicia y lo bueno y como el imperio de la ley apunta a las condiciones por las cuales el derecho puede servir para evitar la injusticia más que a ser un mero instrumento; analiza varios casos judiciales internacionales, entre ellos uno que involucra a Chile, que en nuestros días, reflexiona el autor, parecen, por sobre todo, expresar el predominio de una tolerancia mundial más que un orden jurídico global. El quinto capítulo examina lo que el autor denomina la reconstitución de lo público; vuelve sobre la estrecha relación entre el derecho y la política; analiza la cuestión o dimensión social de la juridificación desde afuera y expone críticamente los modelos de lo público multinivel y la síntesis de la legalidad; destaca la función esencial de lo jurídico en la tarea de instituir y delimitar lo que constituye lo público en un contexto mundial, velando porque se respeten los criterios de la no arbitrariedad, de la no dominación; el comportamiento que no supere el test de la racionalidad, de la proporcionalidad, de la justificación más allá del interés propio, señala el profesor, violaría las condiciones de lo justo, imponiendo sobre el globo una insostenible concesión unilateral del bien. Concluye Palombella que la reconstitución de lo público, a partir de sus componentes abstractos, kantianos, no es más que otra forma de presentación de una opción por el Rule of law a escala mundial.

La premisa fundamental de la que parte el libro es que el derecho público multinivel puede llevarnos a un gobierno mundial, basado en una legalidad global, que surja de una juridicidad horizontal, que sea fruto del trabajo de cortes 
internacionales y nacionales fuertemente vinculadas por lazos de colaboración o cooperación recíprocos, como ocurre en la actualidad en la Unión Europea.

El autor, Palombella, nos sumerge a lo largo de 272 páginas en el mundo de la interacción entre globalización, derecho y gobernanza. Los fenómenos, los problemas y las tendencias de la llamada "globalización" se han transformado en una de las características importantes del mundo en las últimas décadas y como tales han sido crecientemente analizadas por múltiples disciplinas. Algunas de estas tendencias globalizantes se dan en las telecomunicaciones, en los deportes, en el comercio internacional ${ }^{3}$, en los derechos humanos, la energía, los ecosistemas, etc. $(\text { p. } 8)^{4}$.

Crecientemente el Derecho intenta aumentar, y de facto aumenta, la regulación de tales intercambios. Es así que se han desarrollado normativas tales como la Convención Europea de Derechos Humanos, las normas de la Organización Mundial del Comercio, las decisiones del Consejo de Seguridad de las Naciones Unidas, la Convención de las Naciones Unidas sobre el Derecho del Mar, etc. (pp. 12-13).

Según el autor el Rule of law (el mandato o imperio del derecho) es además de tendencial y creciente, deseable y difícil. Ideal y abstractamente considerado, tiende a ser supra o extraestatal. El autor cita, en este sentido, a G. Sartori: "el imperio del derecho no postula al Estado, postula, más bien, un derecho extraestatal, autónomo: el derecho común, la jurisprudencia, en fin el derecho de los jueces y de los juristas" (p. 38). ${ }^{5}$

Esta característica se ha consolidado en el área europea continental sólo en los últimos sesenta años (p.11). Citando a Kelsen, Palombella afirma que este Rule of law neutralizaría las diferencias ético-culturales de los Estados y las sociedades (p. 78). Sin embargo, esto "funciona a lo más como un objetivo, un ideal normativo" (p. 88). ${ }^{6}$

El Rule of law se diferencia del Rule by law (el mandato o imperio por el derecho), de carácter más instrumental, sin que el primero implique necesariamente

\footnotetext{
3 Es importante el ejemplo que da el autor del caso Swordfish, entre la Unión Europea y Chile, el que trata en la p. 167, donde se contraponen intereses provenientes de un derecho supraestatal con intereses amparados por un derecho nacional.

4 Ésta y las otras referencias corresponden a la edición citada.

5 "il Rule of law non postula lo stato, postula, piuttosto, un diritto extrastatuale, autonomo: il diritto comune, il case law, insomma il diritto dei giudici e dei giuristi" (p. 38).

6 "funziona semmai come un obiettivo, un ideale normativo" (p. 88).
} 
concepciones iusnaturalistas (p. 24). No obstante la tendencia al derecho global, más allá del derecho internacional, el autor señala que: "(...) el considerado "derecho global" es sólo uno entre los niveles de legalidad que habitan el globo..." y que "mantener las diferencias, proteger la autonomía, es posible sólo si las relaciones son arbitradas con un principio de equilibrio, de no-dominación" (p. 13) 7 .

La tendencia del Rule of law es, por tanto, la máxima subordinación posible de los poderes transitorios y/o arbitrarios al mandato o imperio de la ley, y no la subordinación de la ley al poder. Sin embargo, dice el autor: "en los hechos -se observa- que el derecho está siempre sometido a la voluntad de la clase dominante (...) En términos abstractos, por lo tanto, es difícil celebrar "El imperio del derecho, no de los hombres" (a saber, tradicionalmente, "el gobierno de las leyes y no de los hombres), de manera del todo inocente. En el ámbito de su espectro semántico, el "gobierno de los hombres" incluye obviamente también el "gobierno por medio del derecho". Esto conduce, justamente, al otro "adversario" del Imperio del derecho, el llamado Imperio por el derecho" (p. 20)8.

Palombella, con referencia a Goodhart (p. 21), piensa que el imperio o mandato de la ley es algo totalmente distinto, ya que mientras el Rule by law puede constituir el medio más eficiente para imponer un gobierno tiránico, el Rule of law indicaría el rule under the law, esto es, el poder bajo la ley, subordinado a la ley, que es el fundamento esencial de la libertad.

Contrasta el autor esta postura con la de autores alternativos, que plantean que esta relación estrecha entre el Derecho (global o no) y el Poder es relativamente inevitable, refiriendo el autor a Stephen Holmes, quien propone sólo una diferencia de grados entre el Rule of law y el Rule by law, agregando, "y la diferencia se debería a la distribución social del poder; ello dependería, en particular, del desarrollo de una plena y completa "poliarquía" (p. 21)9.

\footnotetext{
7 “(...) il cosiddetto "diritto globale" é solo uno tra gli strati di legalitá che abitano il globo...” y que "Mantenere le differenze, proteggerne l'autonomia, é possibile solo se le relazioni siano arbítrate da un principio di bilanciamento, di non-dominazione" (p. 13).

8 "nei fatti -si osserva- il diritto é sempre sottomesso alla volontá della classe dominante (...) In termini astratti, dunque, é difficile celebrare il 'Rule of law, not men' (ossia, tradizionalmente, 'il governo delle leggi e non degli uomini'), in modo del tutto innocente. Nell' ambito del suo spettro semántico, il 'governo degli uomini' include ovviamente anche il 'governo per mezzo del diritto'. Ció conduce, appunto, innanzi all'altro 'avversario' del Rule of law, il cosiddetto Rule by law' (p. 20).

9 "e la differenza sarebbe dovuta alla distribuzione sociale del potere; essa dipenderebbe, in particolare, dallo sviluppo di una piena e completa 'poliarchia' (p. 21).
} 
Análoga tesis se encuentra en Pettit, quien considera que el ideal del Rule of law implica una igual distribución del poder entre los ciudadanos y, por tanto, una democracia neorrepublicana ideal (p. 47).

Una diferencia esencial del derecho global o la vigencia del Rule of law en el mundo, con el Derecho Internacional, es que este último mantiene la predominancia de los Estados: en la expresión anglófona citada por Palombella: "the Rule of law in this jurisdiction" (p. 89).

El proceso más avanzado de una concreción ideal del modelo del Estado de Derecho, la encuentra el autor (como ya señalamos), en la Unión Europea: "el 'estado de derecho' constitucional europeo representa una aproximación avanzada al modelo ideal del imperio del derecho. Conscientes de esto, la Unión Europea llama al imperio del derecho una conquista propia (...) Por tanto, Europa considera al imperio del derecho como un conjunto de parámetros óptimos para el derecho occidental, lo incluye entre los propios 'valores fundamentales', junto a los derechos humanos, a la libertad o a la democracia. Y por lo tanto como un ideal para recomendar, cualquiera sea la extensión que tenga el orden jurídico" (p. 89) ${ }^{10}$.

En contraste, el autor describe diferentes regulaciones relativistas y pragmáticas del "Rule of law... in this jurisdiction", especialmente de Estados Unidos, que emergen en contra de los derechos civiles y políticos de la Convención de Ginebra (pp. 90 y 93) y contra la Corte Internacional de Justicia (p. 251).

Pero el autor critica también, de otro modo, las limitaciones de la propia Unión Europea: "Un segundo rasgo importante en el análisis de Europa ha sido, y lo es todavía, la interpretación de su déficit democrático"... "El único anhelo -y a largo plazo- es que se forme una verdadera cultura política europea (inmediatamente supranacional)"... "cuando cada pueblo debe obedecer las decisiones cuya autoridad no deriva de su exclusiva autonomía sino de la voluntad de un gran número de otros" (p. 239) ${ }^{11}$.

10 "lo 'stato di diritto' costituzionale europeo rappresenta un'approssimazione avanzata al modello ideale del Rule of law. Consapevole di questo, l'Unione Europea si richiama al Rule of law come una conquista propria (...) Pertanto, l'Europa considera il Rule of law come un insieme di parametri ottimali per il diritto occidentale, lo include tra i propri 'valori' fondamentali, accanto ai diritti umani, alla libertá o alla democrazia. E dunque come un ideale da raccomandare, qualunque estensione abbia l'ordine giuridico” (p. 89).

11 "Un secondo importante tratto nell' analisi dell'Europa é stato, ed é ancora, l'interpretazione del suo deficit democratico:"... "L'unico auspicio -e di lungo período- é che si formi una vera cultura política europea (immediatamente sovranazionale)"... "quando ciascun popolo deve obbedire a decisioni la cui autoritá non deriva dalla sua esclusiva autonomia ma dalla volontá di un grande numero di altri” (p. 239). 
En conclusión la respuesta a la pregunta que titula el libro es que una legalidad global es posible, pero no actualmente y en cualquier caso será lenta y difícil. Para el autor es además imprescindible diferenciar globalización y universalidad, lo que también ocurre para todas las ciencias sociales: "no hay necesaria coincidencia entre globalización y iniversalidad (o universabilidad). Ellas no coinciden: una es un dato, la otra un criterio normativo (moral). Una cosa es la extensión de hecho del poder decisional de aquellos regímenes supranacionales, otra son las condiciones normativas (en el sentido no sólo de corrección jurídica, sino que de justicia)" (p. 261) ${ }^{12}$.

Para seguir aproximándonos a este ideal, la comunicación en el mundo deberá hacerse más dialógica y más funcional, disminuyendo los aspectos neoimperiales que suele tener. Al respecto el autor señala: "comunicación significa que las partes pueden ambas aprender una de la otra, pero sólo si el intérprete está autorizado para avanzar su propio argumento (y si lo posee). Aprender es un beneficio esencial de la comunicación, y si ambas partes lo hacen posible es también una medida de corrección"(p. 175) ${ }^{13}$.

Por otra parte, también es necesario, para lograr el objetivo de una legalidad mundial o global, supraestatal, disminuir el etnocentrismo, esto es, no sentir y creer que la cultura propia es mejor que la de otro pueblo, sino que hay que promover mayores grados de respeto de la cultura de todos los pueblos o etnias, considerándolas tan valiosas como la propia, reconociendo nuestras diferencias como legítimas y enriquecedoras.

Palombella, finalmente, en esta línea argumental, nos recuerda algo que es casi de sentido común pero que tendemos a olvidar: "el Imperio del derecho requiere de vínculos de mutuo reconocimiento entre pares, de la racionalidad y la universalidad de los argumentos" (p. 177) ${ }^{14}$.

A mi juicio, lo precedentemente señalado, se consigue, en mayor grado, con regímenes políticos democráticos, donde el derecho no sea considerado un mero

\footnotetext{
12 "non necessaria coincidenza tra globalizzazione e universalitá (o universalizzabilitá). Esse non coincidono: l'una é un dato, l'altra un criterio normativo (morale). Una cosa é l'estensione di fatto globale del potere decisionale di quei regimi sovranazionali, un'altra sono le condizioni normative (in senso non solo di correttezza giuridica, ma di giustizia)” (p. 261).

13 "comunicazione significa che le parti possono entrambe imparare l'una dall'altra, ma solo se l'interprete é autorizzato ad avanzare il suo proprio argomento (e se lo possiede). L'apprendere é un beneficio essenziale della comunicazione, e se reso possibile ad entrambe le parti é anche una misura di correttezza" (p. 175).

14 "il Rule of law richiede vincoli di mutuo riconoscimento tra pari, la razionalitá e l'universalizzabilitá degli argomenti” (p. 177).
} 